\title{
High fidelity 3D thermal nanoimprint with UV curable polydimethyl siloxane stamps
}

\author{
Nachiappan Chidambaram and Robert Kirchner ${ }^{\text {a) }}$ \\ Paul Scherrer Institute, Laboratory for Micro- and Nanotechnology, 5232 Villigen PSI, Switzerland \\ Mirco Altana \\ Heptagon Oy, Moosstrasse 2, 8803 Rüschlikon, Switzerland \\ Helmut Schift \\ Paul Scherrer Institute, Laboratory for Micro- and Nanotechnology, 5232 Villigen PSI, Switzerland
}

(Received 24 June 2016; accepted 5 August 2016; published 19 August 2016)

\begin{abstract}
A two-step replication process chain is developed for a microlens array structure with deep three dimensional (3D) reliefs and sharp features enabling the transfer of a photocured acrylic resist patterns into thermoplastic poly-methyl methacrylate (PMMA) with the same structural polarity via an intermediate stamp. By using ultraviolet (UV)-curable polydimethyl siloxane (PDMS), high fidelity negatives were cast from the original microstructures made by two-photon-polymerization and subsequently replicated into PMMA using thermal imprint. The mechanical properties of the new UV-PDMS (X-34-4184, Shin-Etsu Chemical Company, Ltd.), along with its nearly zero process shrinkage, proved to be highly suitable to replicate both $50 \mu \mathrm{m}$ high concave features and sharp tips with an apex diameter of $500 \mathrm{~nm}$. The results prove that silicone rubber, despite its elasticity, has specific advantages in thermal imprint in structures where both tall microstructures and submicron surface structures have to be replicated. This way, high fidelity PMMA structures with low defects could be prepared by the optimized processing found in this work to have a replication of 3D masters for further upscaling. (C) 2016 American Vacuum Society.
\end{abstract}

[http://dx.doi.org/10.1116/1.4961250]

\section{INTRODUCTION}

Functional polymeric devices, whose predominant cost goes into the design and development of the master microstructure, can be profitably replicated with UV/thermal rollto-roll nanoimprint lithography (NIL), ${ }^{1,2}$ micro/nanoinjection molding, ${ }^{3,4}$ or UV/thermal wafer level $\mathrm{NIL}^{5-7}$ with high throughput. In such an environment, one can allow longer time and/or higher cost for the fabrication of master structure; hence, high resolution lithographic techniques such as electron-beam writing can be employed (for instance, in diffractive optical elements). But for refractive micro-optical elements, direct write laser-lithography (DWL) with single photon absorption [Fig. 1(a)] is the preferred choice for mastering, since writing tall lenses beyond $10 \mu \mathrm{m}$ height are not possible with electron-beam lithography. In DWL with single photon absorption, by varying the dose applied to each pixel on the resist, different heights can be achieved during development, resulting in 3D microstructures (gray-scale patterning). ${ }^{8,9}$ There are no undercuts and hollow features that are possible with gray-scale process, so it might be termed $2.5 \mathrm{D}$ to be more accurate. ${ }^{10}$ Although DWL with gray-scale processing is widely accepted as a well-documented process, ${ }^{11}$ getting desired precision and thickness control is cumbersome, which requires lot of development-inspection cycles and demands highly controlled environment. DWL with two photon polymerization (2PP), i.e., two photon absorption process for negative resist, ${ }^{12}$ provides an alternative to the single photon absorption overcoming the above mentioned issues,

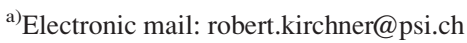

when used in Dip-in mode, where the resist acts as an index matched immersion medium. ${ }^{13}$ Here, an IR laser is used to expose the UV sensitive resist through a high numerical aperture lens [Fig. 1(b)]. The photoreaction only occurs in the region of very high intensity (around the focal spot) where two IR photons are simultaneously absorbed (within few femtoseconds). Since the two photon process is highly nonlinear, resolution is much higher compared to the wavelength used for exposure. When compared to DWL with single photon absorption, two photon process boasts four times better lateral resolution while vertical resolutions are difficult to compare since in a gray-scale process vertical resolution depends on the number of gray levels possible and the thickness of the resist. For a given resist, better resolution equates to better surface finish, but for different resists, proximity effect/photoinitiator diffusion lengths must be considered as well.

Transferring the master onto a working stamp is a very important step in UV/thermal NIL, for the following reasons: multiply the number of replicas of the master structure, easier to handle the negative tone working stamps (important features are embedded in, when master microstructures are protruding out), and is preferred for the harsh replication conditions (often of lower value compared to the master). Ideally, one tried to have a stamp with both hard and elastic properties, ${ }^{14}$ like thin electroplated nickel shims ${ }^{8}$ with high ability to bend. Elasticity can be from the material property or from the geometric parameter (thickness) or both. Quartz is another option where one can have extremely high hardness while settling for lower elasticity (still larger than silicon stamps). But often, when the replication conditions are not as harsh, UV curing hybrid polymer provides a 
(a)

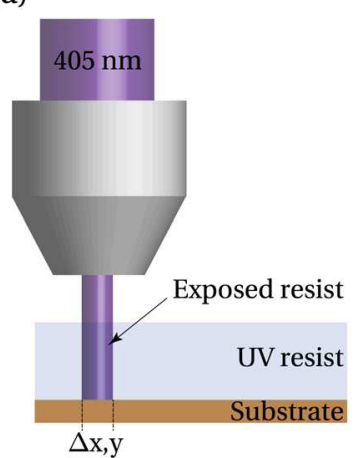

(b)

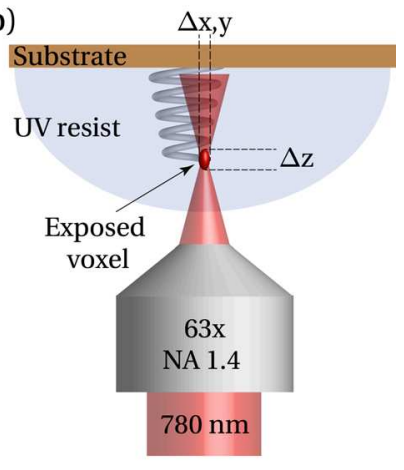

FIG. 1. (Color online) Illustration of (a) 1 photon (UV light) and (b) 2 photon (IR light) absorption process in direct write laser-lithographic technique (DWL).

cost-effective substitute with high hardness but low elasticity. ${ }^{15-17}$ In the other end of spectrum, soft molds made of silicone [polydimethyl siloxane (PDMS)] show interesting possibility and have opened up a whole new field of softlithography. ${ }^{18,19}$ The advantages of soft molds are high defect tolerance (large elongation before failure), ease of using on curved substrates, and replicating features with undercuts ${ }^{20}$ while remaining very low-cost. The multilayered stamp materials are also an attractive option to have tailored stamp properties, like hard surface with an elastic backbone [hard PDMS over soft PDMS (Ref. 21), OrmoStamp ${ }^{\circledR}$ over PDMS (Ref. 22)]. The PDMS stamps are widely accepted for UV NIL where pressures are not very high $(0.01-0.1 \mathrm{MPa})$, but generally refrained to be used in thermal NIL or injection molding due to higher pressure and mold deformation from the highly viscous material being molded. Although there has been several reports on PDMS molds used for thermal NIL, ${ }^{23-26}$ they have been primarily used for cost-effective rapid prototyping and the ease of use on curved substrates. ${ }^{27}$ It was also shown that highly cross linked PDMS with higher strengths are resulting in better pattern replication accuracy compared to softer PDMS and also has longer life time. ${ }^{28,29}$ We have established thermal NIL with UV curable PDMS stamps with high replication fidelity, which also presents huge potential for economical rapid prototyping for roll-to-roll thermal NIL. We also present a viable process chain for the two-step replication of tall microstructures with sharp features. The aim of the process is to provide a method for the transfer of the pattern from $2 \mathrm{PP}$ resist into a material which allows up-scaling (e.g., by step and repeat) and postprocessing (e.g., by exposure with electrons and thermal reflow). Scientifically new in this work is the investigation of the interdependencies of the process parameters imprint pressure, imprint temperature, and

holding time for a faithful replication of high fidelity, sub$200 \mathrm{~nm}$ features in combination with complex and large 3D microstructures. Such an extensive evaluation of the respective process window as well as comparison of different stamp materials is novel in this regard.

\section{EXPERIMENTAL DETAILS}

Master microlens arrays were fabricated on a glass substrate using DWL with 2PP process. For this purpose, commercial tool from Nanoscribe GmbH "Photonic Professional GT" was used in Dip-in mode on high resolution resist from Nanoscribe GmbH "Ip-Dip" [Fig. 2(a)]. In contrast to Fig. 1(b), the objective is immersed into the resist during writing (hence "Dip-in" mode). The master structures were directly written from the design file. The design was sliced due to the required writing discretization in the vertical direction by $0.5 \mu \mathrm{m}$ and in horizontal direction by $0.2 \mu \mathrm{m}$, as the exposed volume element (voxel) is elongated along the optical axis [see Fig. 1(b)]. Seven hundred and eighty nanometers pulsed femtosecond laser was continuously scanned in the in-plane directions using galvo mirrors to write the horizontal slices, and then, piezoelectric actuator was used for the out-of-plane direction to write the 3D microstructure by stacking vertical slices. After each vertical slice was written, the direction of writing was rotated by $45^{\circ}$, so that any in-plane asymmetry is avoided. Then, the surrounding unexposed liquid Ip-Dip resist was developed using propylene glycol monomethyl ether acetate for $15 \mathrm{~min}$. Afterward the master structures were coated with thin layer of antisticking layer, similar to the process explained in Ref. 30. The working stamp was fabricated by drop casting UV curing resist on the master structure followed by front and back side exposure as well as curing [Figs. 2(b)-2(d)]. Three different UV curable stamp materials were considered: Ip-Dip (Nanoscribe $\mathrm{GmbH}$ ), a hard, fully organic, acrylic polymer; OrmoStamp (micro resist technology $\mathrm{GmbH}$ ), a hybrid silicon containing polymer and X-34-4184; and UV-PDMS (Shin-Etsu Chemical Company, Ltd.), a soft elastomeric polymer. This UVPDMS has a higher tensile strength when compared to conventional thermocurable PDMS Sylgard ${ }^{\circledR} 184 .{ }^{31}$ A summary of various materials used here along with its mechanical properties are summarized in Table I. Note that the strength measured under compression is typically higher than the one measured under tension. Additionally, microscale testing is also different from bulk values.

Adhesion between the glass handling wafer and stamp material was improved by pretreatment with oxygen plasma. UV exposure was done with ELC-500 from Electro-Lite

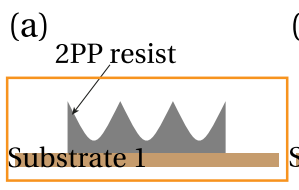

Master structure

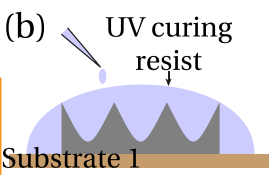

Drop casting

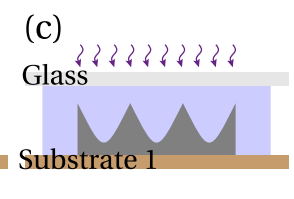

$1^{\text {st }}$ UV exposure (d) Stamp

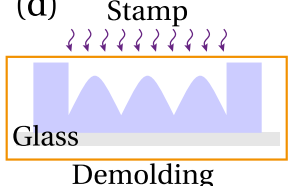

Demolding $\& 2^{\text {nd }}$ exposure

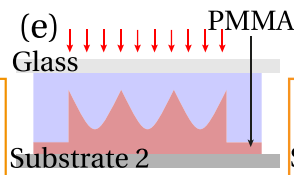

Thermal NIL

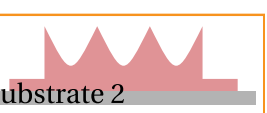

Replica of the master

FIG. 2. (Color online) Schematic of the process flow starting from definition of master structures in Ip-Dip (a), making the negative tone of the master (stamp) onto a UV resist (b)-(d) and replicating the master structure into a thermoplastic by thermal NIL (e)-(f). 
TABLE I. Mechanical properties of various polymeric materials used in this study.

\begin{tabular}{|c|c|c|c|}
\hline Material/property & Strength (MPa) & Elongation to break (\%) & Comment \\
\hline $\begin{array}{l}\text { Standard PDMS (Sylgard 184, } \\
\text { Dow Corning Corporation) }\end{array}$ & 3.5 [tensile (Ref. 32)] & 175 [elastomeric (Ref. 32)] & Soft PDMS with sub- $1 \mu \mathrm{m}$ resolution \\
\hline $\begin{array}{l}\text { UV-PDMS (X-34-4184) } \\
\text { Shin-Etsu Chemical Company, Ltd. }\end{array}$ & 7.7 (tensile) & 110 (elastomeric) & Hard PDMS with sub- $100 \mathrm{~nm}$ resolution \\
\hline $\begin{array}{l}\text { OrmoStamp (micro resist } \\
\text { technology GmbH) }\end{array}$ & Not specified & Not specified (brittle) & $\begin{array}{l}\text { Hybrid organic-inorganic material } \\
\text { with sub- } 100 \mathrm{~nm} \text { resolution }\end{array}$ \\
\hline Ip-Dip (Nanoscribe GmbH) & $\begin{array}{l}90 \text { [microcompressive } \\
\text { (Ref. 33)] }\end{array}$ & $\begin{array}{l}10 \text { [microcompressive, } \\
\text { brittle (Ref. 33)] }\end{array}$ & $\begin{array}{l}\text { Hard acrylic polymer } \\
\text { sub- } 100 \mathrm{~nm} \text { resolution }\end{array}$ \\
\hline PMMA & 50-70 (tensile) & $2-5.5$ (brittle) & Thermoplastic with sub-10 $\mathrm{nm}$ resolution \\
\hline
\end{tabular}

Corporation with the peak intensity at $365 \mathrm{~nm}$. The exposure doses were $18,7.2$, and $5.4 \mathrm{~J} / \mathrm{cm}^{2}$ for Ip-Dip, OrmoStamp, and UV-PDMS, respectively. All these are mildly overexposed compared to the manufacturer's recommendation. All the stamps were coated with antisticking layer after a low power oxygen plasma treatment. Thermal NIL was performed into polymethyl methacrylate (PMMA) film on a glass substrate using HEX 03 press from Jenoptik Mikrotechnik GmbH. PMMA 120k pellets (from micro resist technology $\mathrm{GmbH}$ ) were dissolved in Anisole to a desired concentration so that after spin coating at $3000 \mathrm{rpm}$, followed by soft-bake at $150^{\circ} \mathrm{C}, 10 \mu \mathrm{m}$ film thickness was obtained. Four layers of $10 \mu \mathrm{m}$ PMMA were deposited on the substrate before it can be imprinted. The microstructures were characterized with Zeiss Supra VP55 scanning electron microscope (SEM). The height of the microlens from the base to tip was measured using an optical microscope with a mechanical stage of $1 \mu \mathrm{m}$ resolution.

\section{RESULTS AND DISCUSSION}

The microstructure of the master is shown in Fig. 3(a), in as-written state, from $2 \mathrm{PP}$ process. The image in the bottom column is the higher magnification version, where one could observe the lens apex. Since these were written layer-by- layer, the slicing steps are visible. The main aim would be to replicate this high curvature apex into the thermoplastic for up-scaling. Therefore, the ideal stamp material (Sec. III A), the optimal process window (Secs. III B and III C), as well as in standard processes usually neglected elastic effects need to be considered (Sec. III D).

\section{A. Comparison of stamp materials}

Figures 3(b)-3(d) show the SEM images of the UV cured stamp, drop cast over the master structure with Ip-Dip resist, OrmoStamp, and UV-PDMS, respectively. It can be seen that in both Ip-Dip and OrmoStamp, the tips of the master structure get broken and stay inside the stamp. However, OrmoStamp replicates the fine features better than Ip-Dip and UV-PDMS. But UV-PDMS is the ideal choice as it is very gentle on the sharp tips of the master structure, in addition to preserving the fine details from the master to a sufficient extent [Fig. 3(d)]. Figures 3(e)-3(g) show the SEM pictures of PMMA film imprinted $\left(180^{\circ} \mathrm{C}, 2.5 \mathrm{MPa}\right.$, and $15 \mathrm{~min}$ ) using the stamp materials shown in Figs. 3(b)-3(d), respectively. Given times are always holding times at imprint temperature, but the overall duration includes heating and cooling times (3-5 min). Naturally, the PMMA imprinted with stamps of Ip-Dip and OrmoStamp cannot be
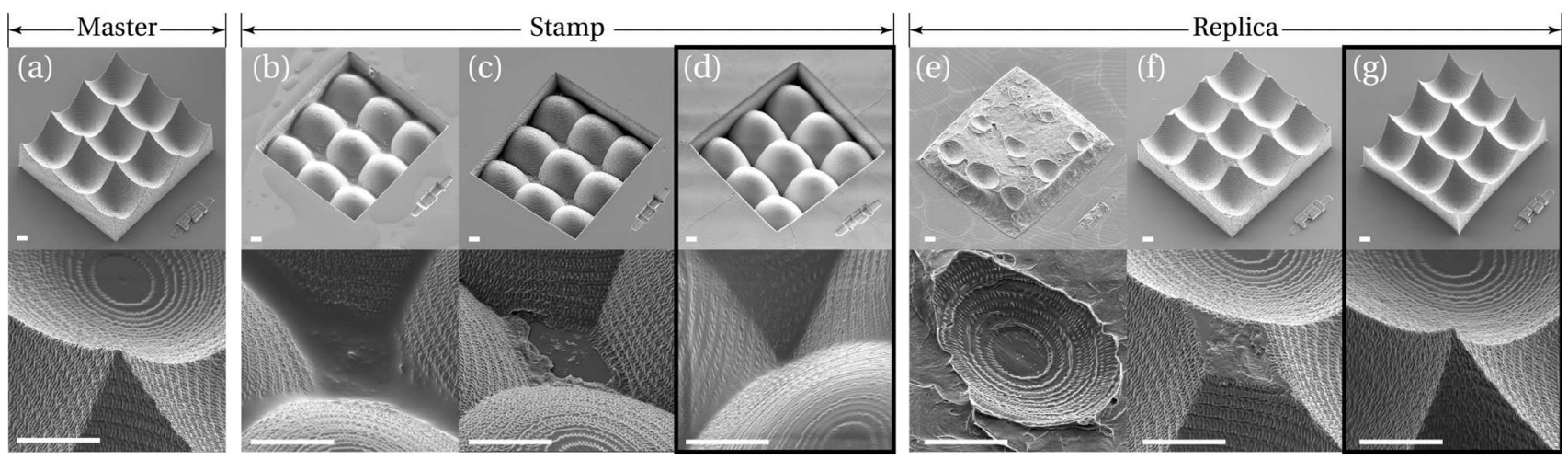

FIG. 3. SEM image of (a) the master fabricated in Ip-Dip using 2PP process, with an array of concave structures with sharp pins, (b)-(d) shows the stamp casted directly from the master with UV curing resist Ip-Dip, OrmoStamp and UV-PDMS, respectively, which shows replicated convex "bumps" with deep trenches between them, (e)-(g) are images of PMMA replica imprinted with Ip-Dip, OrmoStamp, and UV-PDMS stamp, respectively. Images on the bottom row show a higher magnification and the scale bar is always $10 \mu \mathrm{m}$. Highlighted images show the best stamp and replica combination. 


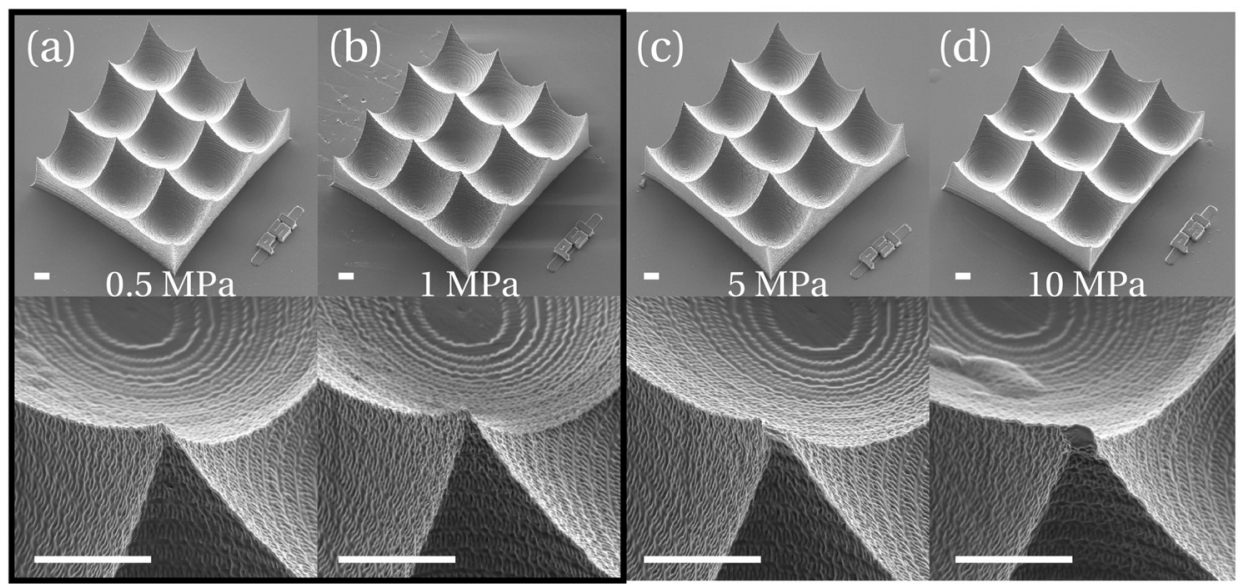

FIG. 4. SEM images of PMMA film imprinted using UV-PDMS stamp, at $180^{\circ} \mathrm{C}$, for $30 \mathrm{~min}$ at various pressures from 0.5 to $10 \mathrm{MPa}$ [(a)-(d)]. Images on the bottom row show a higher magnification and the scale bar is $10 \mu \mathrm{m}$. The optimal imprinting pressure is between 0.5 and $2.5 \mathrm{MPa}$ [cf. also Fig. 3(g)].

completely imprinted since it already has part of the master inside. Ip-Dip tends to rip off PMMA features [Fig. 3(e)] and was not stable in high temperature conditions. The fracture of PMMA into Ip-Dip could be due to the nonelastic deformation of the stamp during thermal NIL which wraps around PMMA features, thereby getting broken during demolding. With the UV-PDMS stamp, many replications were possible and all of them showed good reproduction of the features present in the master structure [compare Figs. 3(a) and 3(g)], attributed mainly due to the elastic nature of the stamp.

\section{B. Process window: Pressure}

Figure 4 shows the SEM images of PMMA imprinted with various imprinting pressures, starting from 0.5 to $10 \mathrm{MPa}\left(180^{\circ} \mathrm{C}, 30 \mathrm{~min}\right)$. It can be seen that even small pressure is sufficient for efficient filling of the mold cavity, in line with the observations of Ref. 25. At extremely high pressures, the tips are broken, but no catastrophic failure like with hard and brittle stamps is observed. This is the inherent advantage of using a very elastic stamp. Although there is slight tip deformation from $5 \mathrm{MPa}$ on, there is a quite large

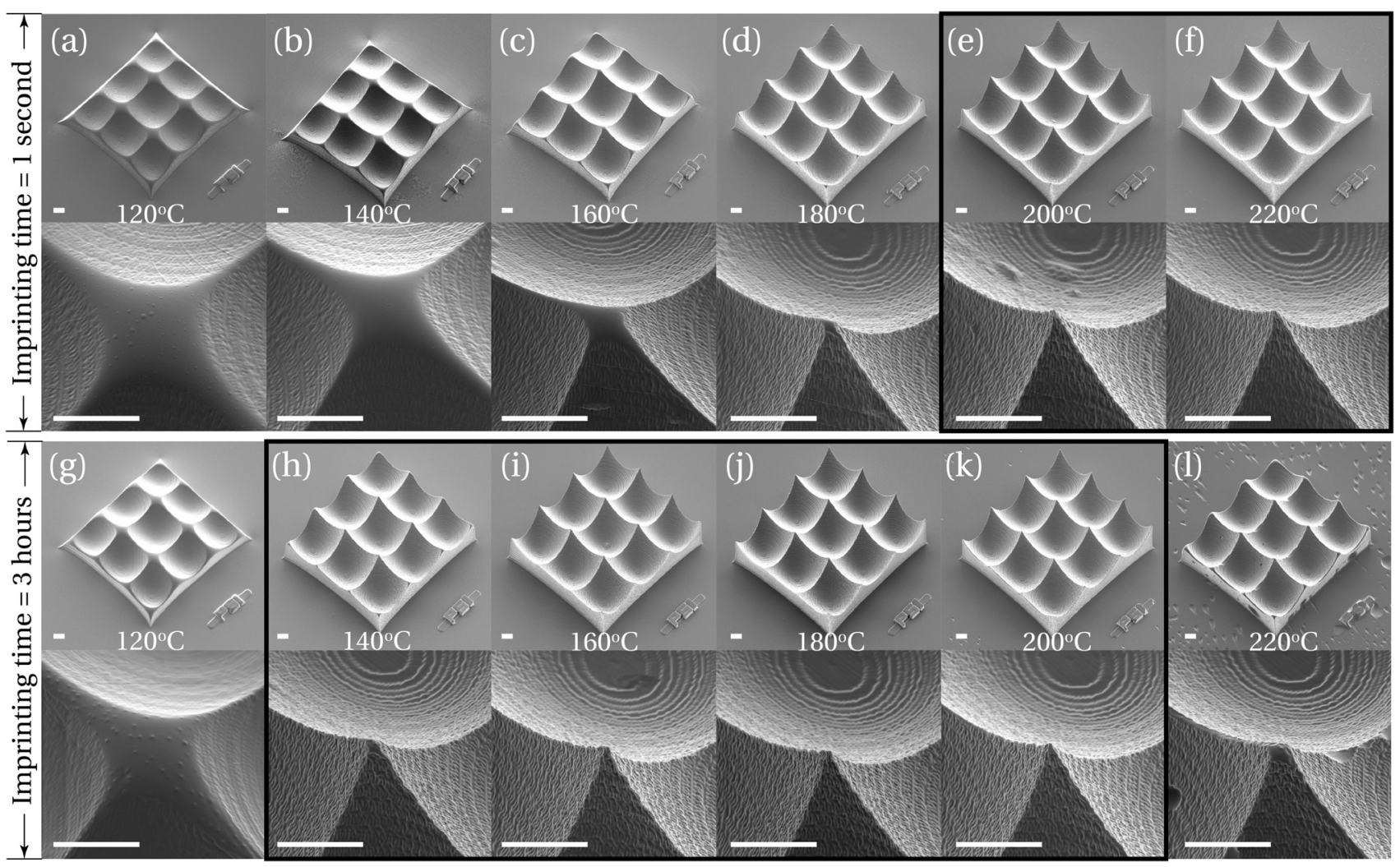

FIG. 5. SEM images of PMMA film imprinted using UV-PDMS stamp, at $120-180^{\circ} \mathrm{C}$, for $1 \mathrm{~s}[(\mathrm{a})-(\mathrm{f})]$ and $3 \mathrm{~h}[(\mathrm{~g})-(\mathrm{l})]$ at $2.5 \mathrm{MPa}$. Images on the bottom row show a higher magnification and the scale bar is $10 \mu \mathrm{m}$. The optimal imprinting temperature for $1 \mathrm{~s}$ imprinting is between 200 and $220^{\circ} \mathrm{C}$ and is between 140 and $200^{\circ} \mathrm{C}$ for $3 \mathrm{~h}$ imprinting. 


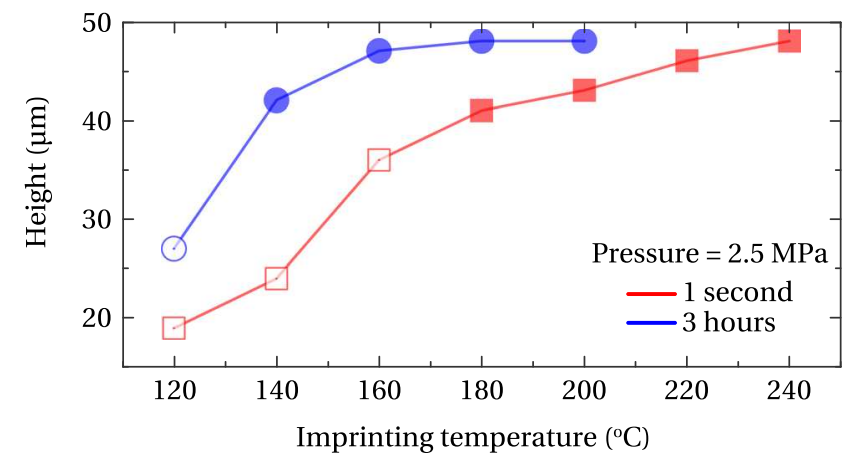

FIG. 6. (Color online) Microlens height as a function of imprinting temperature and time. Solid data points are patterns where PMMA completely fills the mold cavity (observation from SEM images). The designed height of the master is $50 \mu \mathrm{m}$.

process window for high fidelity pattern replication in terms of pressure, with the range between 0.5 and $5 \mathrm{MPa}$.

\section{Process window: Time and temperature}

On top of pressure, one can also work with temperature to tune the viscosity of the imprint polymer. Figure 5 shows the SEM images of PMMA imprinted at different temperatures and imprinting time at $2.5 \mathrm{MPa}$. At low temperature, the PMMA is viscous and so does not allow the complete filling of PDMS mold, thereby leaving partially filled patterns [Figs. 5(a)-5(c) and 5(g)]. For low imprinting times, one needs very high temperature to have complete filling [Figs. 5(e) and 5(f)], which is intuitive. The process window for optimal imprinting is enlarged when the imprinting time is increased. When the PDMS stamp is used at very high temperatures for a long time, there are some out gassing that degrades the quality of replication [Fig. 5(1)].

Figure 6 presents the height of the microstructure (designed for $50 \mu \mathrm{m}$ ) as a function of imprinting time and temperature. This novel finding shows that mold deformation is still present even after complete filling of the mold which is detrimental for the performance of micro-optical elements as realized in this work and needs to be minimized. One can see that it is not a step function but a gradual increase toward $50 \mu \mathrm{m}$, which indicates that the stamp deforms. There are conditions where there is not enough temperature or time for the stamp to elastically relax and recover its initial shape. Good replication observed for $1 \mathrm{~s}$ imprint at $240^{\circ} \mathrm{C}$ and $3 \mathrm{~h}$ imprint at $140^{\circ} \mathrm{C}$ are the extreme case situations. For instance, $240^{\circ} \mathrm{C}$ is already beyond the typical temperatures for both, PMMA and PDMS, but it demonstrates the ability of fast filling if the viscosity is low enough. In contrast to this, imprint times of several hours allows the PMMA to penetrate into narrow trenches and tips in the PMMA mold even at low temperatures. However, at low temperatures, although not seen in the shape of the lenses, viscoelastic PMMA recovery behavior cannot be excluded. Although we only present a limited variety of structures, the wide range of process parameters for optimal microstructures demonstrates the ability to use PDMS as a mold for thermal imprint of complex structures. For this, a process window comprising different combinations of pressure, temperature, and time can be found which can be adapted to different structural designs.

\section{Stamp deformation: Observations and theory}

Figure 7 illustrates the PMMA imprinting process using PDMS molds with increasing times/temperature, both having congeneric effects. Even though being only qualitatively, the following model of deformation has a significant impact on 3D structure replication. At low time/temperatures [Fig. 7(b)], the nominal pressure applied is primarily borne by the large protrusions surrounding the mold cavity, and the pressure inside the mold cavity is almost zero. While casting the viscous PDMS on top of the concave master structures does not present a challenge (replication is primarily limited by the demolding of the sharp pillars), the thermal imprint needs to squeeze the polymer below these protrusions into the mold cavity. At low time/temperatures, the ability of the PMMA to flow is very limited; therefore, the stamp gets compressed (there is lateral expansion with a Poisson's ratio of 0.5 , without any densification) and the cavity is slowly filled. At higher time/temperatures [Fig. 7(c)], the ability of the PMMA to flow is enhanced (especially on the edge between mold cavity and the protrusions); therefore, the stamp can be bent downward in areas where flow is high. ${ }^{34,35}$ At very high time/temperatures [Fig. 7(d)], the flow is so high that the compression is homogenized and so PMMA lens height becomes very close to the designed heights of the master. This implied that there is a time constant above which PDMS recovers its compressive strain (induced by nonhomogeneous stress distribution) and this time depends on the viscosity/temperature of the PMMA. Narasimhan and Papautsky also observed this relaxation time required for thermal NIL of PDMS into thermoplastics. ${ }^{24}$ The compressive strain is more severe in the case where there are no large area protrusions surrounding the (a)

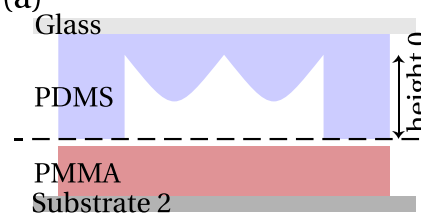

(b)

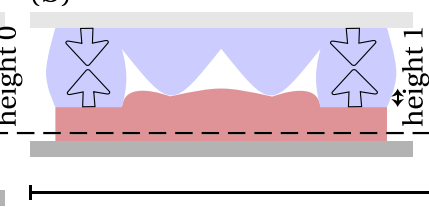

(c)

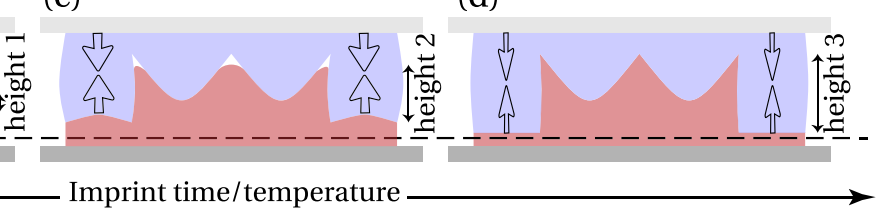

FIG. 7. (Color online) Illustration of thermal nanoimprint using PDMS stamp with increasing imprint time/temperature. At low temperature/time, the pressure distribution is very inhomogeneous, and with increasing time/temperature, the pressure distribution is equilibrated. The width of the open arrow indicates the amount of compressive strain in the vertical direction. The initial height of the mold cavity, marked as "height 0," is larger than the height of replica at lower time/temperature (height $0>$ height $3>$ height $2>$ height 1 ). The deformation and Poisson's ratio effect are magnified for clarity. 
device structures, so very high time/temperature combinations might be required to achieve sufficient strain relaxation.

The use of PDMS as stamp for thermal NIL, although not new, is particularly interesting for applications, as in this work, where delicate structures are required to be molded and demolded. The UV-PDMS from Shin-Etsu Chemical Company, Ltd., is tough enough to replicate high aspect ratio structures and gentle enough to demold inherent roughness and sharp features. There is moderate reduction in roughness during the replication (between master and replica), although beneficial here for the microlens structures, is small enough that a faithful replication down to the sub-50 nm range can be postulated. The stamp survives harsh conditions (temperatures up to $240^{\circ} \mathrm{C}$ and pressures up to $5 \mathrm{MPa}$ ). In the first case, as shown for Sylgard 184 PDMS, it is evident that temperatures above $200{ }^{\circ} \mathrm{C}$ have to be avoided to reduce or avoid degradation and outgassing; in the second case, pressure, it is evident that the compression of stamp protrusions touching the PMMA during imprint and the overall deformation of the stamp has to be reduced or avoided by process and mold design. The current molding of small area, convex mold structures into concave lenses seems, along with the large protrusions surrounding the lens array, to be well suited for this purpose. Overall, the relaxation of the stamp material, leading to a restoration of its initial shape, helps to retain the exact replication of the stamp. For this, a large process window was found in which no major deviations from the original form could be seen. The overall process is, however, dynamic and also dependent on geometrical issues.

\section{CONCLUSION}

The current experiments demonstrate the successful copying of delicate original structures from 2PP exposed acrylic resist into PMMA by using UV-curable PDMS stamps in a thermal imprint process. The process range is large enough that the replication fidelity of both sharp tips of about $500 \mathrm{~nm}$ and the original $50 \mu \mathrm{m}$ height of the lens structure can be retained; the ability to imprint into PMMA demonstrates the possibility to copy originals into specific thermoplastic materials for postprocessing in industrial upscaling processes. Apart from the almost zero shrinkage, the higher tensile strength of UV-PDMS (X-34-4184) in comparison to standard PDMS (Sylgard 184) is high enough to replicate concave microlens structures. The high elasticity and robustness in comparison to OrmoStamp enables the demolding of tall structures with the sub- $200 \mathrm{~nm}$ roughness inherent to 2PP structures with no defects. This shows that the UVcurable PDMS exhibits the capability to replicate microstructures with high resolution and shape fidelity, which can lead to easier industry adoption. Controlling of the elastic mold deformation is important as demonstrated in this work. As long as UV-PDMS is mostly used as intermediate step for mold fabrication, issues like lifetime do not play a major role. Future experiments will show whether the process range of using PDMS molds in thermal NIL can also be proven for high aspect ratio structures and nanostructures.

\section{ACKNOWLEDGMENTS}

The authors thank Swiss Nanoscience Institute, Basel, for funding this work (NanoArgovia Project No. A10.13SurfFlow), micro resist technology $\mathrm{GmbH}$ for providing PMMA 120k pellets, and Konrad Vogelsang for his help with thermal NIL.

${ }^{1}$ F. C. Krebs, T. Tromholt, and M. Jørgensen, Nanoscale 2, 873 (2010).

${ }^{2}$ J. J. Dumond and H. Y. Low, J. Vac. Sci. Technol., B 30, 010801 (2012).

${ }^{3}$ D. Macintyre and S. Thoms, Microelectron. Eng. 41, 211 (1998).

${ }^{4}$ H. Schift, P. Urwyler, P. M. Kristiansen, and J. Gobrecht, J. Micro/ Nanolithogr., MEMS MOEMS 13, 031303 (2014).

${ }^{5}$ S. Y. Chou, P. R. Krauss, and P. J. Renstrom, J. Vac. Sci. Technol., B 14, 4129 (1996)

${ }^{6}$ S. Landis, N. Chaix, C. Gourgon, C. Perret, and T. Leveder, Nanotechnology 17, 2701 (2006).

${ }^{7}$ H. Schmitt, M. Rommel, A. J. Bauer, L. Frey, A. Bich, M. Eisner, R. Voelkel, and M. Hornung, Microelectron. Eng. 87, 1074 (2010).

${ }^{8}$ Y. Hirai, S. Harada, H. Kikuta, Y. Tanaka, M. Okano, S. Isaka, and M. Kobayasi, J. Vac. Sci. Technol., B 20, 2867 (2002).

${ }^{9}$ A. Schleunitz, V. A. Guzenko, M. Messerschmidt, H. Atasoy, R. Kirchner, and H. Schift, Nano Convergence 1, 7 (2014).

${ }^{10}$ H. Schift, Appl. Phys. A 121, 415 (2015).

${ }^{11}$ M. T. Gale and K. Knop, Proc. SPIE 0398, 347 (1983).

${ }^{12}$ S. Maruo, O. Nakamura, and S. Kawata, Opt. Lett. 22, 132 (1997).

${ }^{13}$ T. Bückmann, N. Stenger, M. Kadic, J. Kaschke, A. Frölich, T. Kennerknecht, C. Eberl, M. Thiel, and M. Wegener, Adv. Mater. 24, 2710 (2012).

${ }^{14}$ H. Schift, J. Vac. Sci. Technol., B 26, 458 (2008).

${ }^{15}$ K. H. Haas and H. Wolter, Curr. Opin. Solid State Mater. Sci. 4, 571 (1999).

${ }^{16}$ H. Schift, C. Spreu, M. Saidani, M. Bednarzik, J. Gobrecht, A. Klukowska, F. Reuther, G. Gruetzner, and H. H. Solak, J. Vac. Sci. Technol., B 27, 2846 (2009).

${ }^{17}$ M. Mühlberger et al., Microelectron. Eng. 86, 691 (2009).

${ }^{18}$ Y. Xia and G. M. Whitesides, Annu. Rev. Mater. Sci. 28, 153 (1998).

${ }^{19}$ H. Schmid and B. Michel, Macromolecules 33, 3042 (2000).

${ }^{20}$ N. Bogdanski, H. Schulz, M. Wissen, H. C. Scheer, J. Zajadacz, and K. Zimmer, Microelectron. Eng. 73, 190 (2004).

${ }^{21}$ T. W. Odom, J. C. Love, D. B. Wolfe, K. E. Paul, and G. M. Whitesides, Langmuir 18, 5314 (2002).

${ }^{22}$ M. Papenheim, C. Steinberg, K. Dhima, S. Wang, and H. C. Scheer, J. Vac. Sci. Technol., B 33, 06F601(2015).

${ }^{23}$ A. P. Russo, D. Apoga, N. Dowell, W. Shain, A. M. P. Turner, H. G. Craighead, H. C. Hoch, and J. N. Turner, Biomed. Microdevices 4, 277 (2002).

${ }^{24}$ J. Narasimhan and I. Papautsky, J. Micromech. Microeng. 14, 96 (2004).

${ }^{25}$ C. K. Malek, J. R. Coudevylle, J. C. Jeannot, and R. Duffait, Microsyst. Technol. 13, 475 (2007).

${ }^{26}$ V. N. Goral, Y. C. Hsieh, O. N. Petzold, R. A. Faris, and P. K. Yuen, J. Micromech. Microeng. 21, 017002 (2011).

${ }^{27}$ W. M. Choi and O. O. Park, Nanotechnology 15, 1767 (2004).

${ }^{28}$ H. Schmitt, P. Duempelmann, R. Fader, M. Rommel, A. J. Bauer, L. Frey, M. Brehm, and A. Kraft, Microelectron. Eng. 98, 275 (2012).

${ }^{29}$ M. Kim, B. U. Moon, and C. H. Hidrovo, J. Micromech. Microeng. 23, 095024 (2013).

${ }^{30}$ H. Schift, S. Saxer, S. Park, C. Padeste, U. Pieles, and J. Gobrecht, Nanotechnology 16, S171 (2005).

${ }^{31}$ K. Mogi, Y. Hashimoto, T. Tsukahara, M. Terano, M. Yoshino, and T. Yamamoto, RSC Adv. 5, 10172 (2015).

${ }^{32}$ F. Schneider, T. Fellner, J. Wilde, and U. Wallrabe, J. Micromech. Microeng. 18, 065008 (2008).

${ }^{33}$ L. R. Meza, A. J. Zelhofer, N. Clarke, A. J. Mateos, D. M. Kochmann, and J. R. Greer, Proc. Natl. Acad. Sci. U. S. A. 112, 11502 (2015).

${ }^{34}$ L. J. Heyderman, H. Schift, C. David, J. Gobrecht, and T. Schweizer, Microelectron. Eng. 54, 229 (2000).

${ }^{35}$ S. Merino, A. Retolaza, H. Schift, and V. Trabadelo, Microelectron. Eng. $\mathbf{8 5}, 877$ (2008). 